\title{
BOUNDS FOR THE VARIATIONAL RATIO OF ARBITRARY LINEAR SYSTEMS
}

\author{
J. ERNEST WILKINS, JR.
}

\begin{abstract}
ABSTRACr. Melese-d'Hospital and Wilkins have recently found bounds for the error involved in replacing the maximum temperature in some geometrically simple regions, containing a nonuniformly distributed heat source, by the maximum temperature in the same region containing a uniform heat source. In this paper we present the formal mathematical reasoning in a general framework suitable for other applications.
\end{abstract}

I. Introduction. In a recent paper [1] the authors considered the question of determining bounds for the error involved in replacing the maximum temperature (or the average temperature) in an infinite slab (or infinite cylindrical shell, or spherical shell), containing a nonuniformly distributed heat source and insulated on one surface, by the maximum (or average) temperature in the same region containing a uniformly distributed heat source with the same overall heat generation rate. In order to make the techniques used in that paper more accessible for other applications, we shall in this paper abstract the essential formal process in the reasoning, as distinct from the manipulative, algebraic, and numerical processes pertaining directly to the heat transfer problem. (These latter processes would of course have to be supplied before useful results in any other application could be obtained.)

Hence we shall be interested in an arbitrary linear functional (e.g., the average value) of a response of a system (e.g., its temperature) to a given source (e.g., the heat source). The principal results, derived in §II, are the inequalities (14) and (17) below. These inequalities express bounds for the error in replacing the linear functional for an arbitrary source by the linear functional for a uniform source in terms of a particular measure of the nonuniformity of the source. Other measures of this nonuniformity are discussed briefly in $\S I I I$.

II. The formal process. Consider a physical situation in which a source or input $H(y)$ defined on the interval $(0,1)$ produces a response or

Received by the editors December 20,1969 and, in revised form, August 14, 1970 and June 5, 1972.

AMS (MOS) subject classifications (1969). Primary 2670.

Key words and phrases. Linear systems, error analysis, error bounds.

(c) American Mathematical Society 1973 
output $T(x)$ defined on $(0,1)$. We suppose that $T(x)$ can be related to $H(y)$ by means of an integral kernel $K(x, y)$ so that

$$
T(x)=\int_{0}^{1} K(x, y) H(y) d y .
$$

Assume further that a significant physically interesting quantity $\tau$ can be expressed as a linear functional of $T$, i.e., that there is a function $P(x)$ such that

$$
\tau=\int_{0}^{1} P(x) T(x) d x .
$$

In this paper we shall study the error involved in using as an estimate of $\tau$ the value $\tau^{*}$ corresponding to a uniform source $H^{*}$ equal to an appropriately weighted average value of $H(y)$, i.e.,

$$
H^{*}=\int_{0}^{1} H(y) \omega(y) d y,
$$

in which $\omega(y)$ is a nonnegative function such that

$$
\int_{0}^{1} \omega(y) d y=1
$$

To be precise, we shall determine the least upper and greatest lower bounds for the dimensionless "variational ratio"

$$
Y=\left(1-\tau / \tau^{*}\right) / V,
$$

in which the total variation $V$ of the normalized source $h(y)=H(y) / H^{*}$ is used as a measure of the departure of $H(y)$ from uniformity.

We suppose that $H(y)$ is a nonconstant function in the class $M$ of bounded measurable functions on $(0,1)$, that $K(x, y)$ is measurable on the unit square, that there exists an integrable function $k(y)$ such that $|K(x, y)| \leqq k(y)$, that $P(x)$ is integrable, that $\omega(y)$ is a nonnegative integrable function satisfying (4), and that $\tau^{*} \neq 0$. These hypotheses suffice to insure the existence of $\tau$ and $\tau^{*}$ and to justify the manipulations to be made below. There is no loss of generality in assuming that $H(y)$ is of bounded variation for otherwise $V=\infty, Y=0$.

We observe first that, as a consequence of (1) and (2),

$$
\tau=\int_{0}^{1} F(y) H(y) d y,
$$

in which

$$
F(y)=\int_{0}^{1} P(x) K(x, y) d x
$$


It follows from this relation and (3) that

$$
\tau^{*}=H^{*} \int_{0}^{1} F(y) d y=\int_{0}^{1} F(z) d z \int_{0}^{1} H(y) \omega(y) d y,
$$

and consequently that

in which

$$
\frac{\tau^{*}-\tau}{\tau^{*}}=\int_{0}^{1} G(y) h(y) d y
$$

$$
G(y)=\omega(y)-F(y) / \int_{0}^{1} F(z) d z .
$$

Let the function $L(y)$ be defined so that

$$
L(y)=-\int_{0}^{y} G(z) d z .
$$

Then $L(0)=0, d L / d y=-G(y)$ almost everywhere, and

$$
L(1)=-\int_{0}^{1} G(y) d y=0
$$

by virtue of (4). Since $h(y)$ is of bounded variation, there are bounded increasing functions $p(y)$ and $n(y)$ which vanish when $y=0$ and are such that

$$
h(y)=h(0)+p(y)-n(y), \quad V=p(1)+n(1) .
$$

It then follows from (8) and (11) that

$$
\frac{\tau^{*}-\tau}{\tau^{*}}=\int_{0}^{1} G(y) p(y) d y-\int_{0}^{1} G(y) n(y) d y=p(1) L(\alpha)-n(1) L(\beta)
$$

for some values $\alpha, \beta$ on $(0,1)$, by virtue of the second theorem of the mean for integrals. In view of (5) and (12) we conclude that

$$
-\max _{0 \leqq y \leqq 1}|L(y)| \leqq Y \leqq \max _{0 \leqq y \leqq 1}|L(y)| .
$$

The upper or lower bound can actually be attained if $\left|L\left(y_{0}\right)\right|=\max |L(y)|$, and $h(y)$ is a step function with a jump at the point $y_{0}$, the jump having the same or opposite sign as $L\left(y_{0}\right)$.

If $G(y)$ is continuous, it follows from (11) that $G(y)$ must vanish at least once on the open interval $(0,1)$. If it is true, as a consequence of the particular physical situation, that $G(y)$ vanishes exactly once, then it follows from Rolle's theorem that $L(y)$ cannot vanish at all on $(0,1)$. In fact, the sign of $L(y)$ on $(0,1)$ is equal to the sign of $G(y)$ near the point $y=1$. It then follows from (13) that, if $h(y)$ is monotone, the lower, 
or upper, bound in (14) can be replaced by 0 , if the signs of $L(y)$ and $h^{\prime}(y)$ are the same, or different.

More generally, if $h(y)$ is monotone increasing, then (14) can be replaced by the inequalities

$$
\min _{0 \leqq y \leqq 1} L(y) \leqq Y \leqq \max _{0 \leqq y \leqq 1} L(y)
$$

whether $G(y)$ vanishes more than once or not. Similarly, if $h(y)$ is monotone decreasing, we see that

$$
-\max _{0 \leqq y \leqq 1} L(y) \leqq Y \leqq-\min _{0 \leqq y \leqq 1} L(y) .
$$

If $h(y)$ is convex and not constant, the bounds expressed in (14) can be improved. To see this, suppose first that $h(y)$ has an absolutely continuous derivative $h^{\prime}(y)$. Let $\xi$ be a maximal value such that $0 \leqq \xi \leqq 1, h^{\prime}(y) \leqq 0$ if $0 \leqq y \leqq \xi, h^{\prime}(y) \geqq 0$ if $\xi \leqq y \leqq 1$, and suppose that

$$
K(s, t)=(s-t)^{-1} \int_{t}^{s} L(y) d y .
$$

Then the denominator in equation (5) becomes

$$
\begin{aligned}
-\int_{0}^{\xi} h^{\prime}(y) d y+\int_{\xi}^{1} h^{\prime}(y) d y= & -\xi h^{\prime}(\xi-)+(1-\xi) h^{\prime}(\xi+) \\
& +\int_{0}^{\xi} y h^{\prime \prime}(y) d y+\int_{\xi}^{1}(1-y) h^{\prime \prime}(y) d y .
\end{aligned}
$$

Since $h(y)$ is itself absolutely continuous, it follows from (8) that

$$
\frac{\tau^{*}-\tau}{\tau}=\int_{0}^{1} L(y) h^{\prime}(y) d y,
$$

so that after a similar splitting of the domain of integration and an integration by parts, we see that

$$
\begin{aligned}
\frac{\tau-\tau^{*}}{\tau^{*}}= & \xi K(\xi, 0) h^{\prime}(\xi-)+(1-\xi) K(\xi, 1) h^{\prime}(\xi+) \\
& -\int_{0}^{\xi} y K(y, 0) h^{\prime \prime}(y) d y+\int_{\xi}^{1}(1-y) K(y, 1) h^{\prime \prime}(y) d y .
\end{aligned}
$$

Since $-h^{\prime}(\xi-), h^{\prime}(\xi+)$ and $h^{\prime \prime}(y)$ are all nonnegative, it now follows that

$$
\begin{aligned}
& Y \leqq \max \left[\max _{0 \leqq y \leqq 1}\{-K(y, 0)\}, \max _{0 \leqq y \leqq 1} K(y, 1)\right], \\
& Y \geqq \min \left[\min _{0 \leqq y \leqq 1}\{-K(y, 0)\}, \min _{0 \leqq y \leqq 1} K(y, 1)\right] .
\end{aligned}
$$


The bounds in (17) are in general sharper than the bounds in (14) because $K(y, 0)$ and $K(y, 1)$ are average values of $L(y)$ and $L(y)$ is in general not constant. In fact, $L(y)$ is constant if, and only if, $F(y)$ is proportional to $\omega(y)$ and then $\tau=\tau^{*}$ is independent of $h(y)$.

In the event contemplated earlier that $G(y)$ is continuous and vanishes exactly once, then $L(y)$, and hence $K(y, 0)$ and $K(y, 1)$ also, has a constant nonzero sign on the open interval $(0,1)$. If this sign is positive, then (17) reduces to

$$
-\max _{0 \leqq y \leqq 1} K(y, 0) \leqq Y \leqq \max _{0 \leqq y \leqq 1} K(y, 1),
$$

while if this sign is negative,

$$
\min _{0 \leqq y \leqq 1} K(y, 1) \leqq Y \leqq-\min _{0 \leqq y \leqq 1} K(y, 0)
$$

When $h(y)$ is concave instead of convex the inequalities (17) and (18) remain valid provided the roles of $K(y, 0)$ and $K(y, 1)$ are interchanged.

The inequalities (17) also hold for a nonconstant convex function $h(y)$ whose derivative $h^{\prime}(y)$ is not necessarily absolutely continuous. When $h(y)$ is convex, its derivative exists and is continuous except possibly at the points of a finite or denumerable set. Moreover, $h^{\prime}(y)$ is a monotone nondecreasing function which is integrable on $(0,1)$, and $h(y)$ is absolutely continuous on the open interval $(0,1)$. The function $h(y)$ need not be continuous at the endpoints $y=0$ and $y=1$, but it does have limiting values $h(0+) \leqq h(0)$ and $h(1-) \leqq h(1)$. If the function $h(y)$ is redefined to be $h(0+)$ when $y=0$ and to be $h(1-)$ when $y=1$, the new function is convex and absolutely continuous on the closed interval $[0,1]$. This redefinition does not alter the value of $\left(\tau^{*}-\tau\right) / \tau^{*}$, and does not increase the total variation $V$, since for the original function,

$$
V=h(0)-h(0+)+\int_{0}^{1}\left|h^{\prime}(y)\right| d y+h(1)-h(1-) .
$$

The new function might be constant, but in this case $Y=0$ for the original function. It is, therefore, sufficient to establish (17) when $h(y)$ is convex, absolutely continuous, and not constant on $[0,1]$.

Consider the polygonal approximation

$$
\begin{aligned}
h_{n}(y) & =h_{i n}+(n y-i)\left(h_{i+1, n}-h_{i n}\right), \\
h_{i n} & =h(i / n), \quad i / n \leqq y \leqq(i+1) / n, i=0,1, \cdots, n-1 .
\end{aligned}
$$

It is not difficult to see that $h_{n}^{\prime}(y)$ converges in the mean of order one to $h^{\prime}(y)$ when $h(y)$ is convex and absolutely continuous on $[0,1]$, and hence that $h_{n}(y)$ converges uniformly to $h(y)$, since $h_{n}(0)=h(0)$. Therefore, 
the variational ratio $Y_{n}$ for $h_{n}(y)$ converges to the variational ratio $Y$ for $h(y)$, and so it is sufficient to show that (17) holds for $Y_{n}$.

Choose $i(n)$ such that $h_{i n} \geqq h_{i(n) n}(i=0,1, \cdots, n)$. Then, except at the corners of the polygon $h_{n}(y), h_{n}^{\prime}(y) \leqq 0$ if $0 \leqq y<i(n) / n, h_{n}^{\prime}(y) \geqq 0$ if $i(n) /$ $n<y \leqq 1$. It follows that the total variation $V_{n}$ of $h_{n}(y)$ is

$$
V_{n}=-\sum_{i=0}^{i(n)-1} \int_{i / n}^{(i+1) / n} h_{n}^{\prime}(y) d y+\sum_{i=i(n)}^{n-1} \int_{i / n}^{(i+1) / n} h_{n}^{\prime}(y) d y .
$$

Let $a_{i n}=n\left[h_{i+1, n}-h_{i n}\right]=h_{n}^{\prime}(y)$ when $i / n<y<(i+1) / n$. Then

$$
\begin{aligned}
V_{n}= & -\sum_{i=0}^{i(n)-1} a_{i n}\left(\frac{i+1}{n}-\frac{i}{n}\right)+\sum_{i=i(n)}^{n-1} a_{i n}\left\{\left(1-\frac{i}{n}\right)-\left(1-\frac{i+1}{n}\right)\right\} \\
= & -\sum_{i=1}^{i(n)-1}\left(a_{i-1, n}-a_{i n}\right) \frac{i}{n}-a_{i(n)-1, n} \frac{i(n)}{n} \\
& +\sum_{i=i(n)+1}^{n-1}\left(a_{i n}-a_{i-1, n}\right)\left(1-\frac{i}{n}\right)+a_{i(n) . n}\left(1-\frac{i(n)}{n}\right) .
\end{aligned}
$$

In a similar manner we see that the value of $\left(\tau^{*}-\tau\right) / \tau^{*}$ for $h_{n}$ is

$$
\begin{aligned}
\int_{0}^{1} G(y) h_{n}(y) d y= & \int_{0}^{1} L(y) h_{n}^{\prime}(y) d y \\
= & \sum_{i=0}^{i(n)-1} a_{i n} \int_{i / n}^{(i+1) / n} d\{y K(y, 0)\} \\
& -\sum_{i=i(n)}^{n-1} a_{i n} \int_{i / n}^{(i+1) / n} d\{(1-y) K(y, 1)\} \\
= & \sum_{i=1}^{i(n)-1}\left(a_{i-1, n}-a_{i n}\right) \frac{i}{n} K\left(\frac{i}{n}, 0\right) \\
& +a_{i(n)-1, n} \frac{i(n)}{n} K\left(\frac{i(n)}{n}, 0\right) \\
& +\sum_{i=i(n)+1}^{n-1}\left(a_{i n}-a_{i-1, n}\right)\left(1-\frac{i}{n}\right) K\left(\frac{i}{n}, 1\right) \\
& +a_{i(n) . n}\left(1-\frac{i(n)}{n}\right) K\left(\frac{i(n)}{n}, 1\right) .
\end{aligned}
$$

Upon comparing these last two results and observing that $a_{i n}-a_{i-1, n}$, $a_{i(n), n}$ and $-a_{i(n)-1, n}$ are all nonnegative, we conclude that $Y_{n}$ does in fact satisfy the inequalities (17).

The bounds in (17) are best possible. If the maximum, or minimum, 
described in (17) is $K\left(y_{1}, 1\right)$, it is attained when $h(y)$ is continuous, constant on $\left(0, y_{1}\right)$, and linear on $\left(y_{1}, 1\right)$ with a positive slope. If the maximum, or minimum, described in (17) is $-K\left(y_{1}, 0\right)$, it is attained when $h(y)$ is continuous, linear on $\left(0, y_{1}\right)$ with a negative slope, and constant on $\left(y_{1}, 1\right)$.

III. Other results. The analysis in §II has used the total variation, which for absolutely continuous $h(y)$ may be expressed as $V=\int_{0}^{1}\left|h^{\prime}(y)\right| d y$, as a measure of the nonuniformity of the source $H(y)$. Other nonnegative functionals of $H(y)$ which vanish if, and only if, $H(y)$ is (almost everywhere) constant are the following:

$$
\begin{aligned}
& V_{p}=\left[\int_{0}^{1}\left|h^{\prime}(y)\right|^{p} d y\right]^{1 / p}, \quad 1 \leqq p<+\infty, \\
& V_{\infty}=\underset{\substack{0 \leqq y \leqq 1 \\
\text { l.u.b. }}}{ }\left|h^{\prime}(y)\right| \text {, } \\
& V_{p}^{*}=\left[\int_{0}^{1}|h(y)-1|^{p} d y\right]^{1 / p}, \quad 1 \leqq p<+\infty, \\
& V_{\infty}^{*}=\underset{0 \leqq y \leqq 1}{1 . u . b .}|h(y)-1|, \\
& V^{\prime}=\operatorname{osc} h(y)=\underset{0 \leqq y \leqq 1}{\text { l.u.b. }}\{h(y)\}-\underset{0 \leq \leqq}{\text { g.l.b.b. }}\{h(y)\} .
\end{aligned}
$$

A variational ratio $Y_{p}, Y^{*}$, or $Y^{\prime}$ can now be defined employing each of these measures of nonuniformity of $H(y)$, and bounds can be found for these variational ratios as $H(y)$ ranges over an appropriate class of functions.

If $h(y)$ is absolutely continuous, it follows from (16) and the Hölder inequality that

$$
\left|Y_{p}\right| \leqq\left[\int_{0}^{1}|L(y)|^{q} d y\right]^{1 / q} \quad(1 \leqq p \leqq \infty)
$$

if $p^{-1}+q^{-1}=1$ and the derivative $h^{\prime}(y)$ is in the class $L_{p}$ of functions for which $V_{p}$ is finite. The inequality (19) is true even if $V_{p}=+\infty$ since in this case $Y_{p}=0$. The inequality (14) is the special case $p=1, q=\infty$ of this result.

Moreover, it follows from (8) and (11), and the normalisation of $h(y)$ that

$$
\frac{\tau^{*}-\tau}{\tau^{*}}=\int_{0}^{1}\left\{G(y)-G^{*} \omega(y)\right\}\{h(y)-1\} d y
$$

for any constant $G^{*}$, and hence

$$
\left|Y_{p}^{*}\right| \leqq \min _{G^{*}}\left[\int_{0}^{1}\left|G(y)-G^{*} \omega(y)\right|^{\alpha} d y\right]^{1 / q} \quad(1 \leqq p \leqq \infty)
$$


For the analysis leading to (20) the earlier assumptions should be modified so that $h(y)$ is in $L_{p}$ and $k(y)$ and $\omega(y)$ are in $L_{a}$. When $1<p \leqq \infty$, so that $1 \leqq q<+\infty$, the minimum in (20) is attained when $G^{*}$ is such that

$$
\int_{0}^{1}\left|G(y)-G^{*} \omega(y)\right|^{q-1} \operatorname{sgn}\left[G(y)-G^{*} \omega(y)\right] \omega(y) d y=0 .
$$

If $p=q=2$, this relation is particularly simple, i.e.,

$$
G^{*}=\int_{0}^{1} G(y) \omega(y) d y / \int_{0}^{1} \omega^{2}(y) d y,
$$

but otherwise we have been unable to exhibit the minimizing $G^{*}$ explicitly.

In order to analyze the behavior of $Y^{\prime}$, we revert to the initial assumptions on $h(y), k(y)$ and $\omega(y)$, and introduce the notation,

Then

$$
E_{+} \equiv[y \mid G(y)>0], \quad E_{-} \equiv[y \mid G(y)<0]
$$

$$
\begin{aligned}
\frac{\tau^{*}-\tau}{\tau^{*}} & =\int_{E_{-}} G(y) h(y) d y+\int_{E_{+}} G(y) h(y) d y \\
& \leqq \text { (g.l.b. } h) \int_{E_{-}} G(y) d y+(\text { l.u.b. } h) \int_{E_{+}} G(y) d y \\
& \left.=\text { (g.l.b. } h) \int_{0}^{1} G(y) d y+\text { (l.u.b. } h-\text { g.l.b. } h\right) \int_{E_{+}} G(y) d y \\
& =(\operatorname{osc} h) \int_{E_{+}} G(y) d y,
\end{aligned}
$$

by virtue of equation (11). Similarly,

and consequently

$$
\frac{\tau^{*}-\tau}{\tau^{*}} \geqq(\operatorname{osc} h) \int_{E_{-}} G(y) d y
$$

$$
\int_{E_{-}} G(y) d y \leqq Y^{\prime} \leqq \int_{E_{+}} G(y) d y .
$$

When $1<p \leqq \infty$, equality will hold in (19) if, and only if,

$$
\begin{aligned}
h(y) & =\alpha+\beta \int_{0}^{y}|L(t)|^{\alpha-1} \operatorname{sgn} L(t) d t, \\
\alpha & =1-\beta \int_{0}^{1}|L(t)|^{\alpha-1} \operatorname{sgn} L(t)\left[\int_{t}^{1} \omega(y) d y\right] d t
\end{aligned}
$$

for some constant $\beta$. Equation (23) is required so that $h(y)$ satisfy the 
norming restriction, inherent in its definition, that

$$
\int_{0}^{1} h(y) \omega(y) d y=1 .
$$

Similarly, when $1<p \leqq \infty$, equality will hold in (20) if, and only if,

$$
h(y)=1+\beta\left|G(y)-G^{*} \omega(y)\right|^{\alpha-1} \operatorname{sgn}\left\{G(y)-G^{*} \omega(y)\right\},
$$

for some constant $\beta$, provided $G^{*}$ is chosen to satisfy (21). This function $h(y)$ surely satisfies (24).

When $p=1$, equality cannot occur in (19) for any absolutely continuous $h(y)$. In fact, we showed in $\S I$ that the bound for $\left|Y_{1}\right|$, defined on the class of functions $h(y)$ of bounded variation, is attained when $h(y)$ is a step function with a jump at a point $y_{0}$ where $\left|L\left(y_{0}\right)\right|=\max |L(y)|$, the magnitudes of the two values of $h(y)$ being scaled, if necessary, so that (24) is satisfied.

When $p=1$, equality holds in (20) if, and only if, $\alpha(y) \equiv h(y)-1$ is positive on the set $\Delta_{0}$, negative on the set $\Delta_{1}$, and zero on the complement of $\Delta_{0}+\Delta_{1}$, the sets $\Delta_{s}$ being defined so that

$$
\begin{aligned}
\Delta_{s} & =\left[y \mid G(y)-G_{0}^{*} \omega(y)=(-1)^{s} F\left(G_{0}^{*}\right)\right], \\
F\left(G^{*}\right) & =\text { ess. lub. }\left|G(y)-G^{*} \omega(y)\right| .
\end{aligned}
$$

The function $h(y)$ will be nonconstant and normed if, and only if, meas $\left(\Delta_{0}+\Delta_{1}\right)>0$, and $\int_{\Delta_{0}+\Delta_{1}} \alpha(y) \omega(y) d y=0$. For the functions $G(y)$ and $\omega(y)$ discussed in [1], the sets $\Delta_{s}$ are in fact finite, and in this case equality cannot hold in (20).

The right, or left, hand bound in (22) will be attained if $h(y)$ is a constant $\alpha$ on the set $E_{+}$and a smaller, or larger, constant $\beta$ on the set $E_{-}$, the magnitudes of $\alpha$ and $\beta$ being scaled, if necessary, so that (24) is satisfied.

If $G(y)$ is continuous and vanishes exactly once, say at the point $y_{0}$, the inequality (22) becomes

$$
-\left|L\left(y_{0}\right)\right| \leqq Y^{\prime} \leqq\left|L\left(y_{0}\right)\right|=\max |L(y)|,
$$

so that $Y$ and $Y^{\prime}$ have the same bounds. The interest in this result stems from the observation that $V^{\prime} \leqq V$ obviously, and so $\left|Y^{\prime}\right| \geqq|Y|$.

\section{REFERENCE}

1. G. B. Melese-d'Hospital and J. E. Wilkins, Heat conduction in one-dimensional geometries with nonuniform internal heat generation, Proc. Fifth Internat. Heat Transfer Conference, Versailles, 1970.

Departiment of Physics, Howard University, Washington, D.C. 20001 